\title{
Modeling the Duality Extraction for Linear Programming Models
}

\author{
Muwafaq M. Fendi AlKubaisi ${ }^{1}$ \\ ${ }^{1}$ University of Bahrain, Bahrain \\ Correspondence: Dr. Muwafaq M. Fendi AlKubaisi, University of Bahrain, Bahrain. E-mail: drfendi@hotmail.com
}

Received: September 23, 2013

Accepted: October 11, 2013

Online Published: November 8, 2013

doi:10.5430/ijba.v4n6p90

URL: http://dx.doi.org/10.5430/ijba.v4n6p90

\begin{abstract}
Many operations research (OR) textbooks have not tackled the problem of converting the various types of Linear Programming (LP) models (i.e. Maximization, Minimization and mixed Constraint Model)from its primal form to dual form. Furthermore, little attention has paid for showing how to extract the dual optimal solution. Extracting duality is of great importance when we tackle problems with mixed constraints models. The primary goal of this research is to demonstrate the methodology adopted in extracting all types of dual models and its optimal solution.
\end{abstract}

Keywords: OR, LP, primal, dual, maximization, minimization, mixed constraints models

\section{Introduction}

Duality refers to the association between the two opponent points of view in any LP problem. The two LP problems are so closely related that the optimal solution of one problem automatically yields the optimal solution to another (H.Taha, 2003). From one point of view, one may look to the problem as maximization (Primal) that is if he/she owns a company produces goods and services and have sold them with a margin of profits.

From another point of view, one may see this problem as a minimization problem (Dual) that is, if he/she may think of renting this company and will try to lower its rental price. Ferguson (2005) asserts the relationship between primal and dual LP indicates that the two problems arise from two different perspectives on the same applications. To make this comparison between the two faces of this problem, we need to perform a sensitivity analysis of the two optimal solutions. Therefore, we need the maximization optimal solution and its minimization optimal solution in order to perform the sensitivity analysis.

Unfortunately, none of the (OR) textbook have shown how anyone can deduce the optimal solution from another type of optimal solution especially when the problem is not in its standard form as we will see in the analysis of this research mentioned later on.

\section{The Methodology}

There are three types of Linear Programming models:

- Standard Maximization Model.

- Standard Minimization Model.

- A Mixed Constraints Model.

The following are suggested steps to perform the duality for each type.

\subsection{Standard Maximization Model}

First, in order to convert the primal problem from the standard maximization form to dual minimization form, we must consider the following points:

1- Number of variables of the primal problem will become the number of constraints of the dual problem.

2- Number of constraints of the primal problem will become the number of variables of the dual problem.

3- Model type maximization will become minimization.

4- The Less or equal sign $(\leq)$ will become Greater or equal sign $(\geq)$.

5- The objective function coefficients of the primal will become the Right Hand Side (RHS) of the Dual.

6- The RHS of the primal will become the objective function coefficients of the dual problem. 
To explain the above points, consider the following Linear Programming problem:

$$
\begin{aligned}
\text { MAX Z } Z=2 X_{1}+4 X_{2}+3 X_{3} \\
\text { S.T } 3 X_{1}+4 X_{2}+2 X_{3} \leq 60 \\
2 X_{1}+X_{2}+2 X_{3} \leq 40 \\
X_{1}+3 X_{2}+2 X_{3} \leq 80 \\
X_{1}, X_{2}, X_{3} \geq 0
\end{aligned}
$$

The optimal Maximization solution to this problem is:

\begin{tabular}{ccccccccc}
\hline \multirow{2}{*}{ Basis } & $\mathrm{C}_{\mathrm{ij}}$ & 2 & 4 & 3 & 0 & 0 & 0 & \multirow{2}{*}{ R.H.S } \\
\cline { 2 - 7 } & & $\mathrm{X}_{1}$ & $\mathrm{X}_{2}$ & $\mathrm{X}_{3}$ & $\mathrm{~S}_{1}$ & $\mathrm{~S}_{2}$ & $\mathrm{~S}_{3}$ & \\
\hline $\mathrm{X}_{2}$ & 4 & $1 / 3$ & 1 & 0 & $1 / 3$ & $-1 / 3$ & 0 & $20 / 3$ \\
\hline$X_{3}$ & 3 & $5 / 6$ & 0 & 1 & $-1 / 6$ & $2 / 3$ & 0 & $50 / 3$ \\
\hline $\mathrm{S}_{3}$ & 0 & $-5 / 3$ & 0 & 0 & $-2 / 3$ & $-1 / 3$ & 1 & $80 / 3$ \\
\hline$Z$ & & $23 / 6$ & 4 & 3 & $5 / 6$ & $2 / 3$ & 0 & $230 / 3$ \\
\hline$C-Z$ & & $-11 / 6$ & 0 & 0 & $-5 / 6$ & $-2 / 3$ & 0 & \\
\hline
\end{tabular}

To extract the dual problem, we can follow the points explained above:

Min. $\mathrm{Z}=60 \mathrm{Y}_{1}+40 \mathrm{Y}_{2}+80 \mathrm{Y}_{3}$

$$
\begin{aligned}
& 3 Y_{1}+2 Y_{2}+Y_{3} \geq 2 \\
& 4 Y_{1}+Y_{2}+3 Y_{3} \geq 4 \\
& 2 Y_{1}+2 Y_{2}+2 Y_{3} \geq 3 \\
& Y_{1}, Y_{2}, Y_{3} \geq 0
\end{aligned}
$$

Second: To extract the dual optimal solution we can perform the following steps:

1- The most important required step here is to recognize the basic dual variables that will appear in the dual optimal solution. We can define these basic dual variables, as the variables did not appear in the primal optimal solution. To make the problem clearer, textbooks have recommended that we use for the dual problem and its slack variables different names from what we have used in primal phase. For example if we nominate in the primal problem variables in terms of $\mathrm{X}_{\mathrm{i}}$, we better have the dual variables in terms of $\mathrm{Y}_{\mathrm{i}}$ (for example). In addition, if we were using $\mathrm{S}_{\mathrm{i}}$ for Slack variables in the primal (Maximization), we may find it easy to give the dual surplus variables $\mathrm{S}_{\mathrm{ii}}$.

2- All of the primal decision variables $\left(X_{1}, X_{2}\right.$, and $\left.X_{3}\right)$ must appear in the dual solution as surplus variables $\left(S_{11}, S_{22}\right.$, and $\left.\mathrm{S}_{33}\right)$ and vice versa. [i.e. all slack variables $\left(\mathrm{S}_{1}, \mathrm{~S}_{2}, \mathrm{~S}_{3}\right)$ of the primal problem must appear in the form of decision variables $\left(\mathrm{Y}_{1}, \mathrm{Y}_{2}, \mathrm{Y}_{3}\right)$ in the dual problem]. Therefore we can make a substitutions table to switch between the primal and dual variables as follows:

\begin{tabular}{lllllll}
\hline Primal Problem Variables & $\mathbf{X}_{1}$ & $\mathbf{X}_{2}$ & $\mathbf{X}_{3}$ & $\mathbf{S}_{1}$ & $\mathbf{S}_{2}$ & $\mathbf{S}_{3}$ \\
\hline Dual Problem Variables & $\mathbf{S}_{11}$ & $\mathbf{S}_{22}$ & $\mathbf{S}_{33}$ & $\mathbf{Y}_{1}$ & $\mathbf{Y}_{2}$ & $\mathbf{Y}_{3}$ \\
\hline
\end{tabular}

3- According to optimal solution above, we should be able to nominate the dimensions of the final dual optimal solution table and its basic variables, and decision and surplus variables. We should use all non-basic variables in the optimal solution $\left(\mathbf{X}_{\mathbf{1}}, \mathbf{S 1}\right.$, and $\left.\mathbf{S}_{\mathbf{2}}\right)$ as dual basic variables. This implies that the non-basic variables $\mathbf{X}_{\mathbf{1}}, \mathbf{S}_{\mathbf{1}}, \mathbf{S}_{\mathbf{2}}$ will appear in duality phase as $\left(\mathbf{S}_{11}, \mathbf{Y}_{\mathbf{1}}\right.$, and $\left.\mathbf{Y}_{\mathbf{2}}\right)$.

4-The value of the objective function of the optimal solution of the minimization problem (dual) should be equal to the value of the maximization problem (230/3).

5-We can deduce the RHS values of the dual optimal solution from the C-Z values of the primal optimal solution. If we want to find the RHS of the variable say $\mathrm{Y}_{1}$, we must look at the value in the row of the $\mathrm{C}-\mathrm{Z}$ in primal optimal solution under its equivalent variable value of $\mathrm{S}_{1}$ (according to the substitution table above). We must not forget to 
multiply the resulting values (that appear always with minus sign) by negative sign, as the values of the RHS column must be always $\geq 0$. (i.e. $11 / 6,5 / 6,2 / 3$ )

6- In addition, we can deduce the values of dual optimal solution in the C-Z row from the RHS of the primal optimal solution. To do so, first we know from our optimal solution above that we have three basic variables: $\mathrm{X}_{2}, \mathrm{X}_{3}, \mathrm{~S}_{3}$. These variables values will represent the values of c-z in duality. Using the substitutions table above, these values of primal solution will represent the values of c-z for $\mathrm{S}_{22}, \mathrm{~S}_{33}$, and $\mathrm{Y}_{3}$ respectively. For example, if we try to extract what value of $\mathrm{Y}_{3}$ column in the $\mathrm{C}-\mathrm{Z}$ row of the dual optimal solution, we must look at the primal RHS for the equivalent variable, which is $\mathrm{S}_{3}$ which equals to $(80 / 3)$ and so on for the rest of c-z row values.

7- To determine the $Z$-row values, it is obvious that these values may be calculated as follows: $\mathrm{Z}=\mathrm{C}$ - (c-z). The most confusing numbers are those of the columns belonging to non-basic variables. The way of finding out these numbers is by defining the dimensions of each value that we try to calculate. This means we must find out what are the names of the row and column that are crossing with each other at a specific crossing-point in the solution table that we try to evaluate. After deciding the row and column names, we change the name of row, column and sign with names in respect to substitutions table. For example in order to find dual value for column $\mathrm{S}_{22}$ and row $Y_{1}\left(S_{22} \& Y_{1}\right)$, we should change names and order $Y_{1}=S_{1}$ and $S_{22}=X_{2}$ (i.e. $\left.S_{1} \& X_{2}=-1 / 3\right)$. Therefore, the optimal solution to the minimization problem is:

\begin{tabular}{|c|c|c|c|c|c|c|c|c|c|c|c|}
\hline \multirow{2}{*}{$\begin{array}{c}\text { Basi } \\
\mathbf{s}\end{array}$} & \multirow{2}{*}{$C_{i j}$} & 60 & 40 & 80 & 0 & 0 & $\mathbf{0}$ & $\mathbf{0}$ & $\mathbf{0}$ & 0 & \multirow{2}{*}{$\begin{array}{c}\text { R.H. } \\
\text { S }\end{array}$} \\
\hline & & $Y_{1}$ & $\mathbf{Y}_{2}$ & $\mathbf{Y}_{3}$ & $S_{11}$ & $\mathbf{a}_{1}$ & $S_{22}$ & $\mathbf{a}_{2}$ & $S_{33}$ & $\mathbf{a}_{3}$ & \\
\hline $\mathrm{S}_{11}$ & $\mathbf{0}$ & 0 & $\mathbf{0}$ & $5 / 3$ & 1 & -1 & $-1 / 3$ & $1 / 3$ & $-5 / 6$ & $5 / 6$ & $11 / 6$ \\
\hline $\mathbf{Y}_{1}$ & 60 & 1 & $\overline{0}$ & $2 / 3$ & $\overline{0}$ & $\mathbf{0}$ & $-1 / 3$ & $1 / 3$ & $1 / 6$ & $-1 / 6$ & $5 / 6$ \\
\hline$Y_{2}$ & 40 & 0 & 1 & $1 / 3$ & 0 & 0 & $1 / 3$ & $-1 / 3$ & $-2 / 3$ & $2 / 3$ & $2 / 3$ \\
\hline $\mathbf{Z}$ & & 60 & 4 & $160 / 3$ & $\mathbf{0}$ & 0 & $-20 / 3$ & $20 / 3$ & $-50 / 3$ & $50 / 3$ & $230 / 3$ \\
\hline $\mathrm{C}-\mathrm{Z}$ & & $\mathbf{0}$ & 0 & $80 / 3$ & 0 & 0 & $20 / 3$ & $-20 / 3$ & $50 / 3$ & $-50 / 3$ & \\
\hline
\end{tabular}

8- You may notice in the duality table that there are three artificial columns $\mathrm{a}_{1}, \mathrm{a}_{2}$, and $\mathrm{a}_{3}$ in the above table. We can extract these columns from the dual solution table above by associating each artificial variable name with corresponding surplus variable name above (i.e. variables $S_{11}, S_{22}$, and $S_{33}$ respectively), and copying the values of the corresponding surplus columns but with opposite sign. For example in order to find the coefficients in $\mathrm{a}_{1}$ column, we need to copy the values in column $S_{11}$ and multiply each number by negative sign as shown in the dual solution table above.

\subsection{Standard Minimization Model}

To convert the problem from standard minimization to standard maximization we can consider the following steps:

1- We use the same notations listed above (for standard Maximization model) except that the Model type minimization will become maximization, the Greater or equal sign $(\geq)$ will become Less or equal sign $(\leq)$.

2- In order to extract the maximization optimal solution from the standard minimization optimal solution we should use the RHS values of the primal solution in negative sign as the optimal solution of the maximization model must always end with zero or negative values.

Consider the previous example. Suppose that the primal problem is:

$$
\begin{aligned}
& \text { Min. } Z=60 Y_{1}+40 Y_{2}+80 Y_{3} \\
& \begin{array}{lll}
\text { S.T } & 3 Y_{1}+2 Y_{2}+Y_{3} \geq 2 \\
4 Y_{1}+Y_{2}+3 Y_{3} \geq 4 & 4 \\
2 Y_{1}+2 Y_{2}+2 Y_{3} \geq & 3 \\
& & Y_{1}, Y_{2}, Y_{3} \geq 0
\end{array}
\end{aligned}
$$

Therefore, the dual problem is: 
$\operatorname{MAX} Z=2 X_{1}+4 X_{2}+3 X_{3}$

$$
\begin{aligned}
& \text { S.T } X_{1}+4 X_{2}+2 X_{3} \leq 60 \\
& 2 X_{1}+X_{2}+2 X_{3} \leq 40 \\
& X_{1}+3 X_{2}+2 X_{3} \leq 80 \\
& \\
&
\end{aligned}
$$

\begin{tabular}{|c|c|c|c|c|c|c|c|c|c|c|c|}
\hline \multirow{2}{*}{$\begin{array}{c}\text { Basi } \\
\mathbf{s}\end{array}$} & \multirow{2}{*}{$\mathbf{C}_{\mathrm{ij}}$} & 60 & 40 & 80 & $\overline{0}$ & 0 & $\mathbf{0}$ & $\mathbf{0}$ & 0 & 0 & \multirow{2}{*}{$\begin{array}{c}\text { R.H. } \\
\text { S }\end{array}$} \\
\hline & & $\mathbf{Y}_{1}$ & $\mathbf{Y}_{2}$ & $\mathbf{Y}_{3}$ & $\mathrm{~S}_{11}$ & $\overline{a_{1}}$ & $\mathbf{S}_{22}$ & $\mathbf{a}_{2}$ & $\mathbf{S}_{33}$ & $\mathbf{a}_{3}$ & \\
\hline $\mathrm{S}_{11}$ & $\mathbf{0}$ & $\overline{0}$ & $\mathbf{0}$ & $5 / 3$ & 1 & -1 & $-1 / 3$ & $1 / 3$ & $-5 / 6$ & $5 / 6$ & $11 / 6$ \\
\hline $\mathbf{Y}_{1}$ & 60 & 1 & $\mathbf{0}$ & $2 / 3$ & $\mathbf{0}$ & 0 & $-1 / 3$ & $1 / 3$ & $1 / 6$ & $-1 / 6$ & $5 / 6$ \\
\hline $\mathbf{Y}_{2}$ & 40 & $\overline{0}$ & 1 & $1 / 3$ & $\mathbf{0}$ & 0 & $1 / 3$ & $-1 / 3$ & $-2 / 3$ & $2 / 3$ & $2 / 3$ \\
\hline $\mathbf{Z}$ & & 60 & 4 & $160 / 3$ & $\mathbf{0}$ & 0 & $-20 / 3$ & $20 / 3$ & $-50 / 3$ & $50 / 3$ & $230 / 3$ \\
\hline C-Z & & $\overline{0}$ & $\mathbf{0}$ & $80 / 3$ & 0 & 0 & $20 / 3$ & $-20 / 3$ & $50 / 3$ & $-50 / 3$ & \\
\hline
\end{tabular}

The primal optimal solution is:

We must define our primal and dual variables in the substitution table as follows:

\begin{tabular}{lllllll}
\hline Primal Problem Variables & $\mathbf{Y}_{1}$ & $\mathbf{Y}_{2}$ & $\mathbf{Y}_{3}$ & $\mathbf{S}_{11}$ & $\mathbf{S}_{22}$ & $\mathbf{S}_{33}$ \\
\hline Dual Problem Variables & $\mathrm{S}_{1}$ & $\mathbf{S}_{2}$ & $\mathbf{S}_{3}$ & $\mathbf{X}_{1}$ & $\mathbf{X}_{2}$ & $\mathbf{X}_{3}$ \\
\hline
\end{tabular}

The final dual optimal solution will consist of $X_{2}$ and $X_{3}$ since $S_{22}$ and $S_{33}$ are non-basic variables in the final primal optimal solution. In addition, $\mathrm{S}_{3}$ should appear in the final table since $\mathrm{Y}_{3}$ did not appear in the primal optimal solution as explained in the following table:

\begin{tabular}{ccccccccc}
\hline \multirow{2}{*}{ Basis } & $\mathrm{C}_{\mathrm{ij}}$ & $\mathbf{2}$ & $\mathbf{4}$ & $\mathbf{3}$ & $\mathbf{0}$ & $\mathbf{0}$ & $\mathbf{0}$ & \multirow{2}{*}{ R.H.S } \\
\cline { 2 - 7 } & & $\mathrm{X}_{1}$ & $\mathrm{X}_{2}$ & $\mathrm{X}_{3}$ & $\mathrm{~S}_{1}$ & $\mathrm{~S}_{2}$ & $\mathrm{~S}_{3}$ & \\
\hline $\mathrm{X}_{2}$ & 4 & $1 / 3$ & 1 & 0 & $1 / 3$ & $-1 / 3$ & 0 & $20 / 3$ \\
\hline $\mathrm{X}_{3}$ & 3 & $5 / 6$ & 0 & 1 & $-1 / 6$ & $2 / 3$ & 0 & $50 / 3$ \\
\hline $\mathrm{S}_{3}$ & 0 & $-5 / 3$ & 0 & 0 & $-2 / 3$ & $-1 / 3$ & 1 & $80 / 3$ \\
\hline$Z$ & & $23 / 6$ & 4 & 3 & $5 / 6$ & $2 / 3$ & 0 & $230 / 3$ \\
\hline $\mathrm{C}-\mathrm{Z}$ & & $-11 / 6$ & 0 & 0 & $-5 / 6$ & $-2 / 3$ & 0 & \\
\hline
\end{tabular}

We can notice that the three artificial columns in the primal optimal solution disappeared in the dual maximization, as we do not need the artificial variables for the standard maximization. In addition, we can notice that we need to multiply the primal RHS with -1 when we use them for the $\mathrm{C}-\mathrm{Z}$ of the dual maximization optimal solution.

\subsection{Mixed Constraints Model (Maximization or Minimization)}

This third type is more complex than the two previous ones, Since it the three types of constraints $\geq$, $\leq$, = and we cannot apply the duality roll of changing the direction of all constraints to opposite since they are not complying with standard form of maximization nor of the minimization. There is a very reliable way to deal with this situation. It is simply converting any problem to standard form problem (i.e. convert all constraints to $\geq$ if we have minimization primal, and convert all constraints to $\leq$ if we have minimization primal) as follows:

1- Put the problem into its standard form by changing the direction of the constraints that do not comply with the type of standard primal LP model by multiplying the whole constraint with -1 .

2- In case that we may face equality constraints $(=)$ in any type of problems, we must split the constraint into two constraints by substitute the equality sign once with $\leq$ sign and once again with $\geq$.(Anderson 2009). The $\geq$ sign 
constraint will not be suitable for the maximization problem therefore we should multiply this constraint with -1 to convert it into $\leq$ constraint. Also, the $\leq$ sign constraint will not be suitable for the minimization problem therefore we should multiply this constraint with -1 to convert it into $\geq$ constraint.

3- Apply the same steps we used in solving standard maximization or standard minimization depending on the type of conversion we have applied as illustrated in the following example.

Suppose we have a minimization problem (not in a standard form). We need to solve this problem as a primal problem and find its dual problem (maximization) and its optimal solution.

Consider the following example:

$\operatorname{Min} \mathbf{Z}=\mathbf{2} \mathbf{X}_{\mathbf{1}}-\mathbf{3} \mathbf{X}_{2}$

S.T:

$\mathrm{X}_{1}+2 \mathrm{X}_{2} \leq 12$

$4 \mathrm{X}_{1}-2 \mathrm{X}_{2} \geq 3$

$6 \mathrm{X}_{1}-\mathrm{X}_{2}=10$

$\mathrm{X}_{1}, \mathrm{X}_{2} \geq 0$

\section{Standard Minimization}

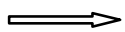

$\operatorname{Min} Z=2 X_{1}-3 X_{2}$

S.T:

$-\mathrm{X}_{1}-\mathrm{X}_{2} \geq-12$

$4 \mathrm{X}_{1}-2 \mathrm{X}_{2} \geq 3$

$\Longrightarrow\left(\begin{array}{l}6 \mathrm{X} 1-\mathrm{X} 2 \geq 10 \\ 6 \mathrm{X} 1-\mathrm{X} 2 \leq 10\end{array}\right) \Longrightarrow\left(\begin{array}{l}6 \mathrm{X} 1-\mathrm{X} 2 \geq 10 \\ -6 \mathrm{X} 1+\mathrm{X} 2 \geq-10\end{array}\right)$

$\mathrm{X}_{1}, \mathrm{X}_{2} \geq 0$

The optimal solution to this problem (primal) is as follows:

\begin{tabular}{ccccccccc}
\hline \multirow{2}{*}{ Basis } & \multirow{2}{*}{$\mathbf{C}_{\mathbf{i j}}$} & $\mathbf{2}$ & $\mathbf{- 3}$ & $\mathbf{0}$ & $\mathbf{0}$ & $\mathbf{0}$ & $\mathbf{0}$ & \multirow{2}{*}{ R.H.S } \\
\cline { 3 - 8 } & & $\mathbf{X}_{\mathbf{1}}$ & $\mathbf{X}_{\mathbf{2}}$ & $\mathbf{S}_{\mathbf{1}}$ & $\mathbf{S}_{\mathbf{2}}$ & $\mathbf{a}_{\mathbf{2}}$ & $\mathbf{a}_{\mathbf{3}}$ & \\
\hline $\mathbf{S}_{\mathbf{1}}$ & $\mathbf{0}$ & 0 & 0 & 1 & -1.625 & 1.625 & -1.25 & 4.375 \\
\hline $\mathbf{X}_{\mathbf{1}}$ & $\mathbf{2}$ & 1 & 0 & 0 & 0.125 & -0.125 & 0.25 & 2.125 \\
\hline $\mathbf{X}_{\mathbf{2}}$ & $\mathbf{- 3}$ & 0 & 1 & 0 & 0.75 & -0.75 & 0.5 & 2.75 \\
\hline $\mathbf{Z}$ & & $\mathbf{2}$ & $\mathbf{- 3}$ & $\mathbf{0}$ & $\mathbf{- 2}$ & $\mathbf{- 2}$ & $\mathbf{1}$ & $\mathbf{- 4}$ \\
\hline $\mathbf{C}-\mathbf{Z}$ & & $\mathbf{0}$ & $\mathbf{0}$ & $\mathbf{0}$ & $\mathbf{2}$ & $\mathbf{2}$ & $\mathbf{- 1}$ & \\
\hline
\end{tabular}

\section{Dual Problem:}

Since in primal optimal solution $\mathrm{X}_{1}$ and $\mathrm{X}_{2}$ were basics that implies in dual phase we will not see any surplus variables in the basis. This would leave only $\mathrm{Y}_{1}$ and $\mathrm{Y}_{3}$ to be basics in dual phase.

\begin{tabular}{ccccccc}
\hline Primal Problem Variables & $\mathbf{X}_{1}$ & $\mathbf{X}_{2}$ & $\mathbf{S}_{1}$ & $\mathbf{S}_{2}$ & $\mathbf{S}_{3}$ & $\mathbf{S}_{4}$ \\
\hline Dual Problem Variables & $\mathbf{S}_{11}$ & $\mathbf{S}_{22}$ & $\mathbf{Y}_{1}$ & $\mathbf{Y}_{2}$ & $\mathbf{Y}_{3}^{\prime}$ & $\mathbf{Y}_{3}$
\end{tabular}

$\operatorname{Max} Z=-12 Y_{1}+3 Y_{2}+10 Y_{3}^{\prime}-10 Y_{3}^{\prime \prime}$

s.t:

$-Y_{1}+4 Y_{2}+6 Y^{\prime}{ }_{3}-6 Y^{\prime \prime}{ }_{3} \leq 2$

$-2 Y_{1}-2 Y_{2}-Y_{3}^{\prime}+Y^{\prime \prime}{ }_{3} \leq-3$ i.e. $\Longrightarrow 2 Y_{1}+2 Y_{2}+Y^{\prime}{ }_{3}-Y^{\prime \prime}{ }_{3} \geq 3$

$Y_{1}, Y_{2} \geq 0 \quad Y_{3}$ unrestricted

The Dual Optimal Solution is:

\begin{tabular}{cccccccccc}
\hline \multirow{2}{*}{ Basis } & \multirow{2}{*}{$\mathbf{C}_{\mathrm{ij}}$} & $\mathbf{- 1 2}$ & $\mathbf{3}$ & $\mathbf{- 1 0}$ & $\mathbf{1 0}$ & $\mathbf{0}$ & $\mathbf{0}$ & $\mathbf{0}$ & \multirow{2}{*}{$\mathbf{R H S}$} \\
\cline { 3 - 8 } & & $\mathbf{Y}_{\mathbf{1}}$ & $\mathbf{Y}_{\mathbf{2}}$ & $\mathbf{Y}_{\mathbf{3}}^{\prime}$ & $\mathbf{Y}^{\prime \prime}$ & $\mathbf{S}_{\mathbf{1 1}}$ & $\mathbf{S}_{\mathbf{2 2}}$ & $\mathbf{a}_{22}$ & \\
\hline $\mathbf{Y}_{\mathbf{2}}$ & 3 & 1.625 & 1 & 0 & 0 & -0.125 & -0.75 & 0.75 & 2 \\
\hline $\mathbf{Y}_{\mathbf{3}}$ & -10 & 1.25 & 0 & 1 & -1 & -0.25 & -0.5 & 0.5 & 1 \\
\hline $\mathbf{Z}$ & & $\mathbf{- 7 . 6 2 5}$ & $\mathbf{3}$ & $\mathbf{- 1 0}$ & $\mathbf{1 0}$ & $\mathbf{2 . 1 2 5}$ & $\mathbf{2 . 7 5}$ & $\mathbf{- 2 . 7 5}$ & $\mathbf{- 4}$ \\
\hline $\mathrm{C}-\mathrm{Z}$ & & $\mathbf{- 4 . 3 7 5}$ & $\mathbf{0}$ & $\mathbf{0}$ & $\mathbf{0}$ & $\mathbf{- 2 . 1 2 5}$ & $\mathbf{- 2 . 7 5}$ & $\mathbf{2 . 7 5}$ & \\
\hline
\end{tabular}


You can notice that if we have a greater constraint then we will have Si \& ai columns in the final table and this is what second constraint in the dual phase above looks like. These columns, as explained earlier, luckily have the same numbers but with different signs. The best to do here is to define the values of $S_{i}$ column then extract the $a_{i}$ column changing the $\mathrm{S}_{\mathrm{i}}$ column to opposite signs.

Finally, we should explain how to determine the coefficient value of basic variable $Y_{3}$ in the above optimal solution table. Is it 10 or -10 as the value of $Y_{3}$ is unrestricted? The simplest way to find out the coefficient of $Y_{3}$ is by forcing the multiplication product of RHS column and the basic variables coefficients (leaving out the $\mathrm{Y}_{3}$ coefficient unknown) to be equal to the objective value as explained below.

$(3 * 2)+\left(\mathrm{Y}_{3} * 1\right)=-4 \quad \longrightarrow \quad \mathrm{Y}_{3}=-10$

Now we can validate the methodology adopted in this paper by extracting the dual of the dual, which should be always primal (Wayne L. Winston 2004). Extraction of the dual of dual will result the following model:

Min $\mathbf{Z}=\mathbf{2} \mathbf{X}_{1}-\mathbf{3} \mathbf{X}_{2}$

s.t:

$-X_{1}-2 X_{2} \geq-12 \quad \Longrightarrow \quad X 1+2 X 2 \leq 12$

$4 X_{1}-2 X_{2} \geq 3$

$6 X_{1}-X_{2}=10$

$\mathrm{X}_{1}, \mathrm{X}_{2} \geq \mathbf{0}$

The model above (dual of the dual) is exactly same primal model we used at the beginning of extraction. We can also prove the dual optimal solution of the dual problem is primal optimal solution using substitution table:

\begin{tabular}{ccccccccc}
\hline \multirow{2}{*}{ Basis } & \multirow{2}{*}{$\mathbf{C}_{\mathbf{i j}}$} & $\mathbf{2}$ & $\mathbf{- 3}$ & $\mathbf{0}$ & $\mathbf{0}$ & $\mathbf{0}$ & $\mathbf{0}$ & \multirow{2}{*}{ R.H.S } \\
\cline { 3 - 8 } & & $\mathbf{X}_{\mathbf{1}}$ & $\mathbf{X}_{\mathbf{2}}$ & $\mathbf{S}_{\mathbf{1}}$ & $\mathbf{S}_{\mathbf{2}}$ & $\mathbf{a}_{\mathbf{2}}$ & $\mathbf{a}_{\mathbf{3}}$ & \\
\hline $\mathbf{S}_{\mathbf{1}}$ & $\mathbf{0}$ & 0 & 0 & 1 & -1.625 & 1.625 & -1.25 & 4.375 \\
\hline $\mathbf{X}_{\mathbf{1}}$ & $\mathbf{2}$ & 1 & 0 & 0 & 0.125 & -0.125 & 0.25 & 2.125 \\
\hline $\mathbf{X}_{\mathbf{2}}$ & $\mathbf{- 3}$ & 0 & 1 & 0 & 0.75 & -0.75 & 0.5 & 2.75 \\
\hline $\mathbf{Z}$ & & $\mathbf{2}$ & $\mathbf{- 3}$ & $\mathbf{0}$ & $\mathbf{- 2}$ & $\mathbf{- 2}$ & $\mathbf{1}$ & $\mathbf{- 4}$ \\
\hline $\mathbf{C}-\mathbf{Z}$ & & $\mathbf{0}$ & $\mathbf{0}$ & $\mathbf{0}$ & $\mathbf{2}$ & $\mathbf{2}$ & $\mathbf{- 1}$ & \\
\hline
\end{tabular}

\section{Conclusion}

1- The steps that the research has demonstrated in the analysis showed step by step that how we could convert the primal model of any type of problem to the dual model of other type (Max \& Min).

2- In addition, we can extract any optimal solution (dual) from any optimal solution (primal) no matter what type of problem we have in hand.

3- primal form of the problem in all types of problems and especially the complex one (mixed constraints) was solved then resolve the dual form in order to see if two solution compatible. The results showed that both forms gave the same optimal solution. This would imply that the arrangements we have adopted to the primal and dual problems were correct.

\section{References}

Ferguson T.S.. (2005). Interpretation of Linear Programming Duality. Retrieved from www.usa.edu.extratebon Hamdy A.TAHA. (2003). Operations Research: An Introduction ( $7^{\text {th }}$ ed.). Prentice Hall - USA.

Jeffrey D. Camm, David Anderson, Thomas Arthur Williams, \& Dennis Sweeney. (2011). An Introduction to Management Science. Thomson-South-Westren, USA.

Marie- France Derhy. (2011). Linear Programming, Sensitivity Analysis \& Related Topics. Prentice Hall, UK.

Mik Wisniewski. (2013). Quantitative Methods for Decision Makers. Prentice Hall, UK.

Wayne L. Winston. (2004). Operations Research Applications and Algorithms. Brooks/Cole- Thomson Learning, USA. 